\title{
THE EFFECT OF A ONE-ON-ONE DIALOGUE-BASED MATHEMATICAL INTELLIGENT TUTORING SYSTEM FOR LEARNING EQUIVALENT FRACTION
}

\author{
Shu Chuan Shih, Hao Yu Tsai, \& Mei Ling Chen \\ Graduate Institute of Educational Information and Measurement, National Taichung University of \\ Education (Taiwan)
}

\begin{abstract}
The purposes of this study are to develop a one-on-one dialogue-based mathematical intelligent tutoring system (ITS) for learning equivalent fraction in the 4th grade math, and evaluate its learning effect. The system used the course content and dialogue script designed by the math teaching experts in advance, and a computer agent teacher asked questions based on the course script. After the student answered, the system was able to identify the error pattern and misconception according to the student's response, then provided each student with adaptive teaching guidance or feedback. Students could construct correct equivalent fraction concepts through a series of interactive dialogues between students and the computer agent teacher step by step. In order to evaluate the effectiveness of this ITS, a quasi-experiment design was conducted. The pretest and post-test were parallel tests involving equivalent fraction. The participants of the study were 76 students in the fourth grade of two elementary schools chosen from midland of Taiwan. They were divided into the experimental group of 39 and the control group of 37 . The experimental group used the "one-on-one dialogue-based mathematical intelligent tutoring system" for teaching. The control group used traditional classroom instruction by a human teacher. The learning content and time were controlled to be the same. Finally, the learning effectiveness and learning interest were assessed by comparing the pre-test and post-test performance of students. The results of the study showed that both teaching methods can significantly improve the students' learning achievements of equivalent fraction, and the learning effectiveness of "one-on-one dialogue-based mathematical intelligent tutoring system" was significantly better than that of traditional classroom instruction. In the "one-on-one dialogue-based mathematical intelligent tutoring system" group, the learning improvement of students with different genders and different ability levels were also reaching a significant level. It indicated that this system benefited the learning achievements of students with different genders and different abilities. Furthermore, from the response data of the learning interest questionnaire, both teaching methods could significantly improve the learning interest of students. But there was no significant difference between the two teaching methods. By interviewing students, the probable causes included that low learning interest students of the experimental group also lack interest to familiar system operation, and some students think this ITS is not interesting enough because of lacking learning games.
\end{abstract}

Keywords: One-on-one, dialogue-based mathematical intelligent tutoring system, basic concepts of fraction, learning effect.

\section{Introduction}

An intelligent tutoring system (ITS) is a computer-assisted tutoring program powered by artificial intelligence (AI). A meta-analysis (Kulik \& Fletcher, 2016) showed that ITSs can be very effective instructional tools for improving students' learning. In particular, one type of ITS is dialogue-based ITS, in which the computer agent has synthesized speech, facial expression in addition to displaying text, graphics, and animation in normal ITSs. The interaction between the speaking agent and learners makes the learning in an ITS more similar to that in the real classroom (Latham et al., 2012). After decades of development, ITSs have been applied in a large spectrum of application, but there are few ITSs for mathematics learning in literature. It can be seen that the development of a math ITS faces more challenges. 


\section{The purpose of this study}

The purposes of this study are to develop a one-on-one dialogue-based mathematical intelligent tutoring system (ITS) for learning equivalent fraction in the 4th grade math, and evaluate its learning effect by a quasi-experiment on 4 th graders in Taiwan.

\section{Method}

In order to evaluate the effectiveness of this ITS, a quasi-experiment design was conducted.

\subsection{The dialogue-based math ITS developed in this study}

The one-on-one dialogue-based mathematical intelligent tutoring system (ITS) for learning equivalent fraction used a talking head at the user interface (UI) to interact with the user. Figure. 1 shows the UI of the math ITS. The tutoring program starts with a main question, which is displayed in the upper left corner. Concurrently, the agent in the upper right corner gives the learner a message about the main question. In the lower left corner is the response area, in which the learner enters input using the keyboard and mouse. After the learner's response has been analyzed, the agent provides encouraging feedback or guidance depending on whether the response is correct or not. The interaction continues until the learner has successfully solved the main question or until he/she fails to solve it, in which case a completed instructional video is presented.

Figure 1. UI of the math ITS.

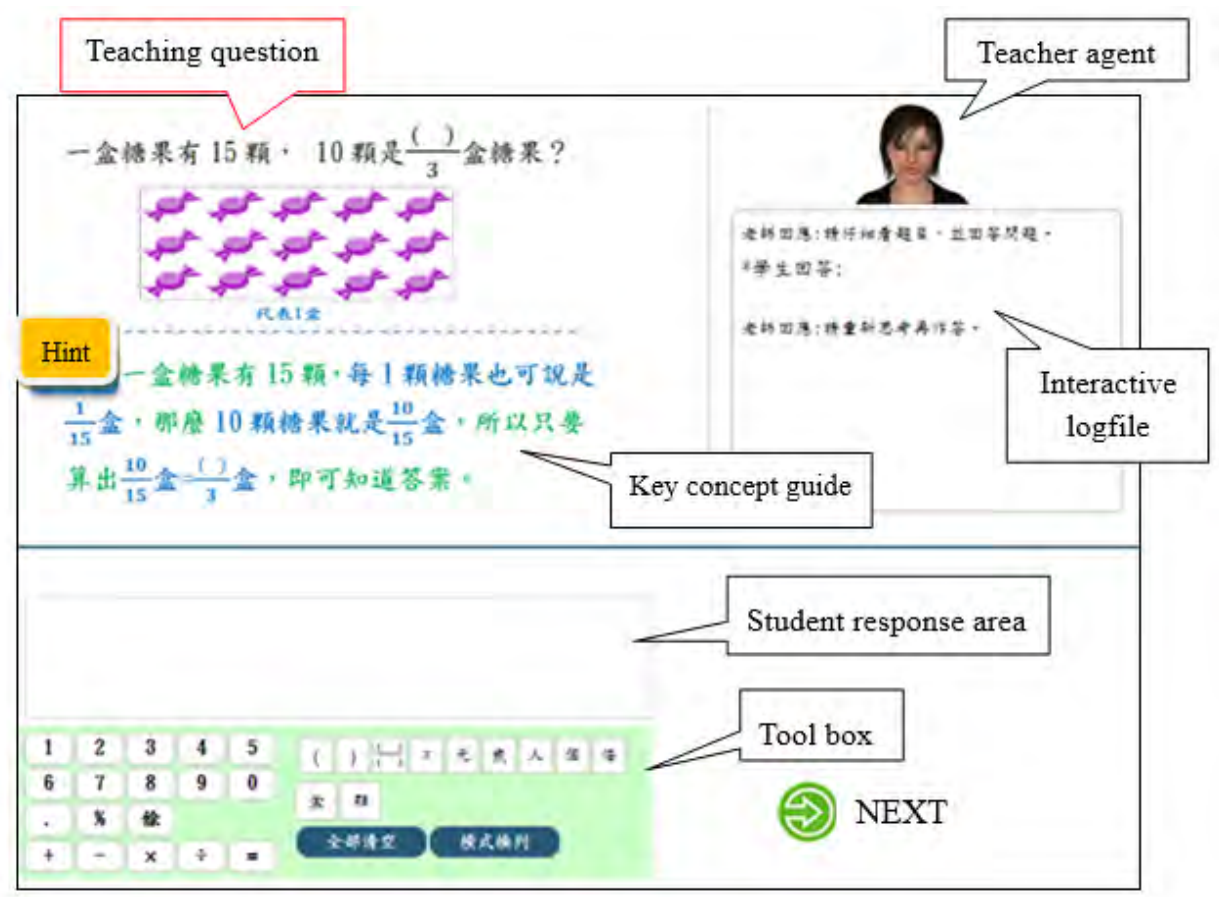

\subsection{Experimental design and participants}

This study used a quasi-experimental design (Figure. 2), the pretest and post-test were parallel tests involving equivalent fraction. Further, the learning interest questionnaire was also conducted before and after the teaching intervention. The participants of the study were 76 students in the fourth grade of two elementary schools chosen from midland of Taiwan. They were divided into the experimental group of 39 and the control group of 37. The experimental group used the "one-on-one dialogue-based mathematical intelligent tutoring system" for teaching. The control group used traditional classroom instruction by a human teacher. The learning content and time were controlled to be the same, and learning time is two hours. Finally, the learning effectiveness and learning interest were assessed by comparing the pre-test and post-test performance of students. 
Figure 2. Experimental design.

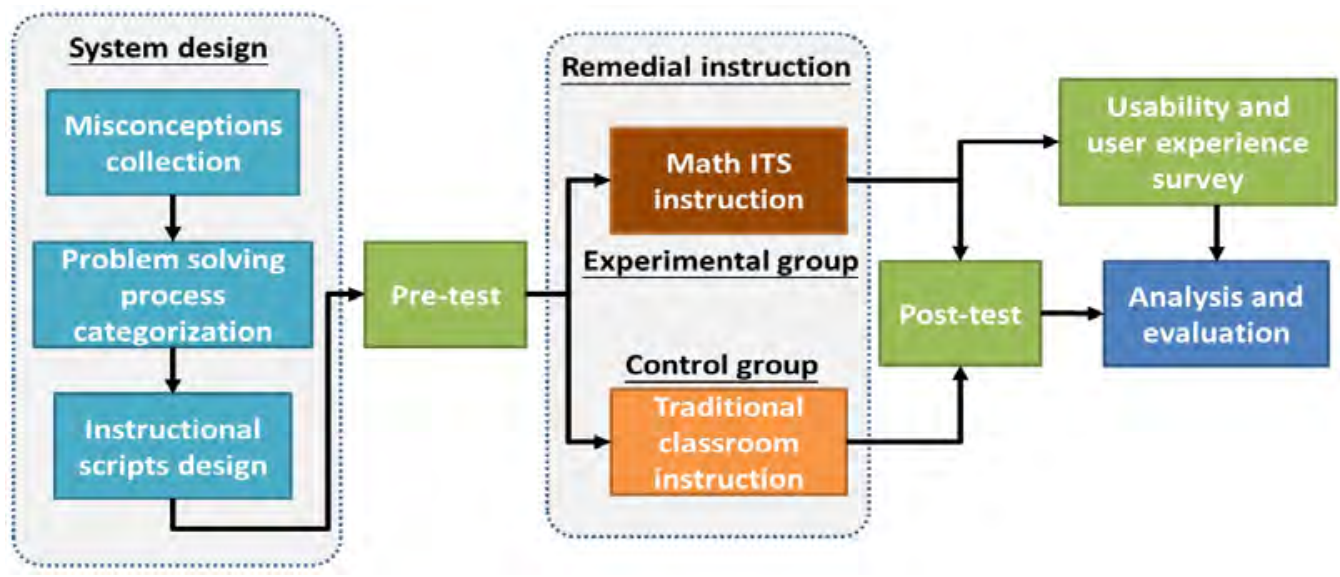

\section{Result}

The results of the study showed that both teaching methods can significantly improve the students' learning achievements of equivalent fraction, and the learning effectiveness of "one-on-one dialogue-based mathematical intelligent tutoring system" was significantly better than that of traditional classroom instruction. In the "one-on-one dialogue-based mathematical intelligent tutoring system" group, the learning improvement of students with different genders and different ability levels were also reaching a significant level. It indicated that this system benefited the learning achievements of students with different genders and different abilities. Furthermore, from the response data of the learning interest questionnaire, both teaching methods could significantly improve the learning interest of students. But there was no significant difference between the two teaching methods. By interviewing students, the probable causes included that low learning interest students of the experimental group also lack interest to familiar system operation, and some students think this ITS is not interesting enough because of lacking learning games.

\section{References}

Efuwape, B. M., \& Aremu, A. (2013). Gender differences in acceptability and usability of computer based learning package in electrical and electronics technology in Nigeria. American Journal of Educational Research, 1(10), 419-424.

Kulik, J. A. \& Fletcher, J. D. (2016). Effectiveness of intelligent tutoring systems: a meta-analytic review. Review of Educational Research, 86(1), 42-78.

Latham, A., Crockett, K., McLean, D., \& Edmonds, B. (2012). Adaptive tutoring in an intelligent conversational agent system. In Transactions on Computational Collective Intelligence VIII (pp. 148-167). Springer, Berlin, Heidelberg. 